\title{
A Continuous Wave Sonic Anemometer-Thermometer ${ }^{1}$
}

\author{
J. C. Katmal ${ }^{2}$ and J. A. Businger \\ University of Washington
}

(Manuscript received 5 March 1962)

\begin{abstract}
A sonic anemometer-thermometer, based on transmission of continuous sound waves and determination of phase angle variations, has been developed. The instrument is designed to measure the fluctuations in the vertical wind component and temperature simultaneously. Measurement of these two quantities enables one to determine the eddy heat flux in the turbulent boundary layer. Field tests indicate that the instrument performs satisfactorily.
\end{abstract}

\section{Introduction}

Experimental investigations of the structure of turbulence and the mechanisms underlying turbulent transport processes in the atmosphere badly need improved instruments that are capable of measuring simultaneously fluctuations of wind, temperature, and concentration of transferable quantities that may be of interest. For measurement of wind and temperature fluctuations, the use of sound waves shows great promise. The attractive characteristics of a sonic anemometer-thermometer (namely, the well-defined path of observation, the simultaneous observation of temperature and wind over the same path, the short response time and the linearity of the response) have focused attention on its development in recent years.

Credit for the first sonic anemometer is given to Carrier and Carlson ${ }^{3}$ who described a true air speed indicator for use on a blimp. Wind velocity was obtained by measuring the phase difference between the signals received by two microphones located upwind and downwind, respectively, from a continuous source of sound. The instrument was never completed. Barret and Suomi (1949) have described a sonic thermometer in which temperature is computed from transit time of sound pulses in air. Corby (1950) has described an anemometer using a single sound source and four microphones placed equidistant at the four cardinal points of a compass. Instruments based on the same method have been described by Schotland (1955), and more recently by Bovsheverov and Voronov (1960) in the U.S.S.R. A

\footnotetext{
1 Contribution No. 73, Department of Atmospheric Sciences, University of Washington, Seattle.

2 Present affiliation: Air Force Cambridge Research Laboratories, Bedford, Mass.

${ }^{8}$ Carrier and Carlson, 1944: A true air speed indicator. NDRC Report, Cruft Laboratories, Harvard University.
}

very small instrument, the "acoustic microanemometer," which has been used to measure the vertical wind component, has been developed by Gurvich (1959).

The most successful attempt at developing a combined sonic anemometer-thermometer was made by Suomi. ${ }^{4}$ In his instrument, velocity and temperature fluctuations were obtained by measuring the difference and sum of transit times for two series of sound pulses traveling in opposite directions along a well-defined path.

The sonic anemometer-thermometer described in this paper is based on the same general principles as the Suomi instrument but instead of sound pulses, continuous signals of two different frequencies are used for transmission in the two directions. Since phase detection can be made independently of amplitude variations in the received signal, the continuous wave system is inherently more stable and less noisy than the pulse system. Phase shifts in the two signals received at either end of the acoustic array are measured accurately; these are then applied to a summing network which performs the appropriate subtraction and addition to yield voltage analogs of the wind component and temperature fluctuations.

\section{Theory of the sonic anemometer-thermometer}

In this section we shall follow rather closely the simple theory described by Suomi and Businger. ${ }^{5}$ Fig. 1 illustrates the effect of wind on the sound ray vectors for a hypothetical case. The sketch shows the transducers

4 Suomi, V. E., 1957: Energy budget studies and development of the sonic anemometer for spectrum analysis. AFCRC Tech. Rep. 56-274, Contract No. AF19(122) -461, University of Wisconsin, Dept. of Meteorology, $91 \mathrm{pp}$.

${ }^{5}$ Suomi, V. E., and J. A. Businger, 1959: Principle of the sonic anemometer-thermometer. AFCRC Tech. Rep. 58-235. Geophysical Research Papers No. 59, p. 1. 


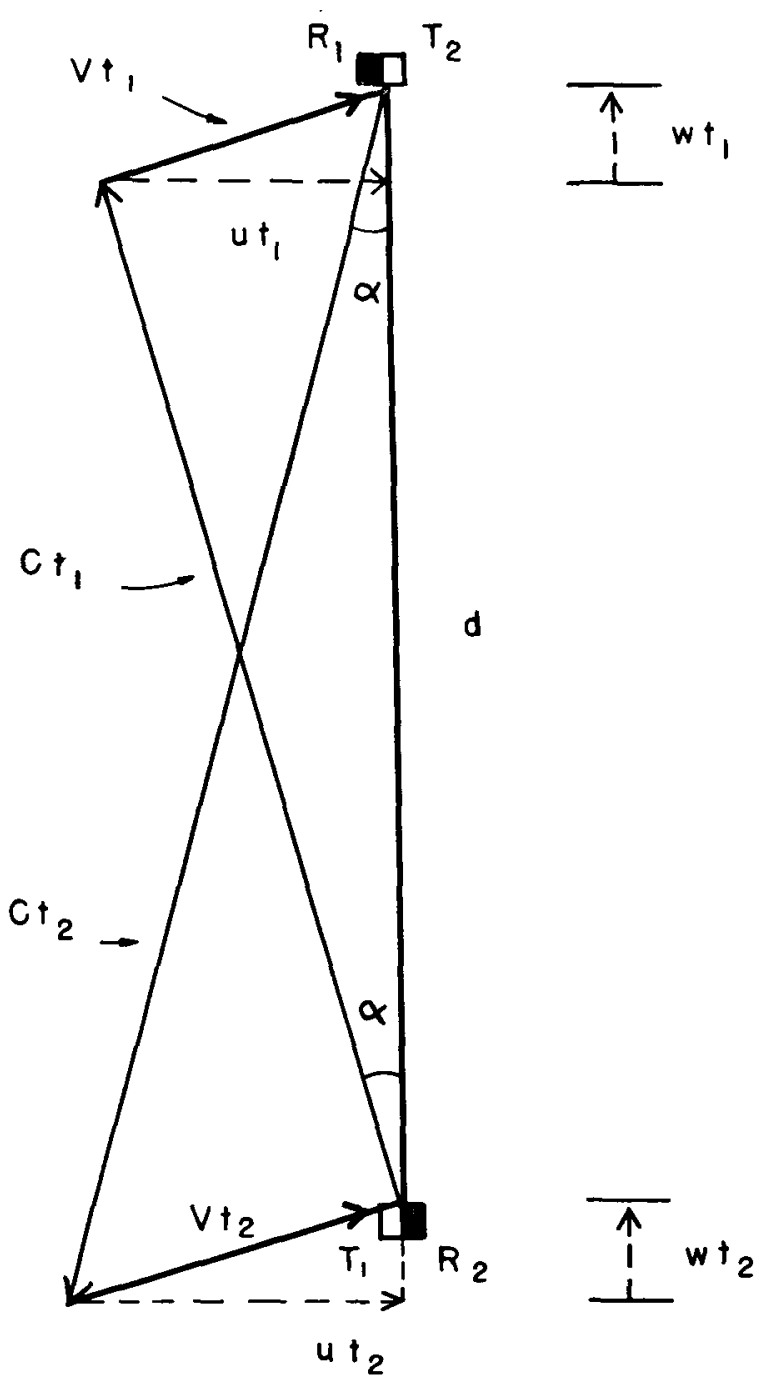

FIG. 1. Sound ray vectors showing the principle of sonic anemometer-thermometer operation.

oriented for measuring the vertical component of the wind because this component is particularly suitable for observation by the sonic anemometer. Sound waves of frequency $f_{1}$ travel upward from the transmitter $T_{1}$ to the receiver $R_{1}$ and waves of frequency $f_{2}$ travel downward from transmitter $T_{2}$ to receiver $R_{2}$.

Assuming that the wind vector $V$ has a component $u$ in the horizontal and $w$ in the vertical, the transit time for a wave front traveling from $T_{1}$ to $R_{1}$ is

$$
t_{1}=\frac{d}{C \cos \alpha+w},
$$

where $d$ is the distance between $T_{1}$ and $R_{1}, C$ the speed of sound in calm air, and $\alpha$ the deviation of the path caused by the horizontal component of the wind. Similarly, the transit time for a wave front from $T_{2}$ to $R_{2}$ is

$$
t_{2}=\frac{d}{C \cos \alpha-\vartheta}
$$

From the relations $V^{2}=u^{2}+w^{2}$ and $\sin \alpha=u / C$ it follows that

$$
\begin{aligned}
& t_{2}-t_{1}=\frac{2 d w}{C^{2}-V^{2}} \\
& t_{2}+t_{1}=\frac{2 d C \cos \alpha}{C^{2}-V^{2}} .
\end{aligned}
$$

$\mathrm{Eq}(4)$ is independent of $w$ and provides the means for measuring temperature. According to theory

$$
C=20.067[T(1+0.32 e / p)]^{1 / 2},
$$

where $T$ is the absolute temperature of the air, $e$ is the vapor pressure of water, and $p$ the atmospheric pressure.

The important variables in (5) can be separated into their mean and fluctuating components as follows:

$$
\begin{array}{r}
\bar{C}+C^{\prime}=20.067(\bar{T})^{1 / 2}(1+0.16 \bar{e} / p) \\
.\left(1+\frac{T^{\prime}}{2 \bar{T}}+\frac{0.16 e^{\prime} / p}{1+0.16 \bar{e} / p}\right) .
\end{array}
$$

Subtracting the equation for the mean components yields the following relationship between the fluctuating components.

$$
\begin{aligned}
C^{\prime} & =\bar{C}\left[\frac{T^{\prime}}{2 \bar{T}}+\frac{0.16 e^{\prime} / p}{1+0.16 \bar{e} / p}\right] \\
& \simeq \bar{C}\left[\frac{T^{\prime}}{2 \bar{T}}+0.16 e^{\prime} / p\right] .
\end{aligned}
$$

Using (7) and neglecting higher order terms, the fluctuating parts of (3) and (4) can be expressed by

$$
\begin{aligned}
& t_{2}{ }^{\prime}-t_{1}{ }^{\prime}=\frac{2 d w w^{\prime}}{\bar{C}^{2}} \\
& t_{2}{ }^{\prime}+t_{1}{ }^{\prime}=-\frac{2 d}{\bar{C}}\left[\frac{T^{\prime}}{2 \bar{T}}+\frac{0.16 e^{\prime}}{p}-\frac{\bar{u} u^{\prime}}{\bar{C}^{2}}\right] .
\end{aligned}
$$

While $\left(t_{2}^{\prime}-t_{1}^{\prime}\right)$ is dependent on $w^{\prime}$ alone, $\left(t_{2}{ }^{\prime}+t_{1}^{\prime}\right)$ depends on $T^{\prime}, e^{\prime}$, and $u^{\prime}$. The effect of $u^{\prime}$ in (9) is intuitively obvious because this component will cause curvature of the sound path. Since there is no clear correlation between $u^{\prime}$ and $T^{\prime}$, the temperature data from this instrument will be useful only when 


$$
\left|\frac{T^{\prime}}{2 \bar{T}}\right| \gg\left|\frac{\bar{u} u^{\prime}}{\bar{C}^{2}}\right| .
$$

For measurement over dry land the term $0.16 e^{\prime} / p$ can be neglected, but over wet ground or over water this term becomes significant and may be corrected for if the Bowen Ratio is known. If a high degree of correlation is assumed between $T^{\prime}$ and $e^{\prime}$, it can be shown that the Bowen Ratio, $\beta$ is approximately equal to $0.68 T^{\prime} / e^{\prime}$. For light winds, (9) may be expressed by

$$
t_{2}{ }^{\prime}+t_{1}^{\prime} \simeq-\frac{d T^{\prime}}{\bar{C} \bar{T}}\left[1+\frac{0.218 \bar{T}}{\beta p}\right] .
$$

The fluctuations in transit time can be expressed in terms of phase fluctuations by putting

$$
t_{1}{ }^{\prime}=-\frac{\phi_{1}{ }^{\prime}}{2 \pi f_{1}}, \quad \text { and } \quad t_{2}^{\prime}=-\frac{\phi_{2}{ }^{\prime}}{2 \pi f_{2}} .
$$

Assuming light winds and dry air, (8) and (9) may be written as

$$
\begin{aligned}
& \phi_{2}{ }^{\prime}-K \phi_{1}{ }^{\prime}=-\frac{4 \pi K f_{1} d w^{\prime}}{\bar{C}^{2}} \\
& \phi_{2}{ }^{\prime}+K \phi_{1}{ }^{\prime}=\frac{2 \pi K f_{1} d T^{\prime}}{\bar{C} \bar{T}} .
\end{aligned}
$$

Here $K=f_{2} / f_{1}$, the ratio of the two frequencies used. The choice of $K$ is a matter of convenience, but since it is necessary to prevent interference between the two channels, identical as well as harmonic frequencies must be avoided. For optimum results $K$ should be as close to unity as possible, depending on the selectivity of the receiver circuit in each channel.

The choice of $d$ and $f_{1}$ is largely dictated by the sensitivity desired and the size of the smallest turbulent eddy one wishes to measure. The sensitivity increases with both $d$ and $f_{1}$ but this is obtained at the expense of total range due to the limit on phase shift that the instrument can indicate without ambiguity.

It is desirable to keep $d$ small enough so that no significant feature on the high-frequency end of the eddy spectrum is lost, but large enough to insure that turbulence generated by the transducers in the acoustic array does not seriously affect the operation of the instrument. It thus becomes obvious that the choice of constants for the sonic anemometer-thermometer is largely a matter of compromise. For this instrument $K=0.75$, and $f_{1}=10 \mathrm{kc}$. For $d=1 \mathrm{~m}$ the total range is is approximately $\pm 5 \mathrm{~m} \mathrm{sec}^{-1}$ on the $w^{\prime}$ scale and $\pm 10 \mathrm{C}$ on the $T^{\prime}$ scale; for $d=50 \mathrm{~cm}$, the range is twice as much.

\section{Description of the instrument}

The sonic aneometer-thermometer (henceforth abbreviated S. A. T.) consists of a single-axis acoustic array and a rack-mounted unit housing all the electronic components (see Fig. 2). The acoustic array has two transmitters and two receivers as in Fig. 1. The elec-

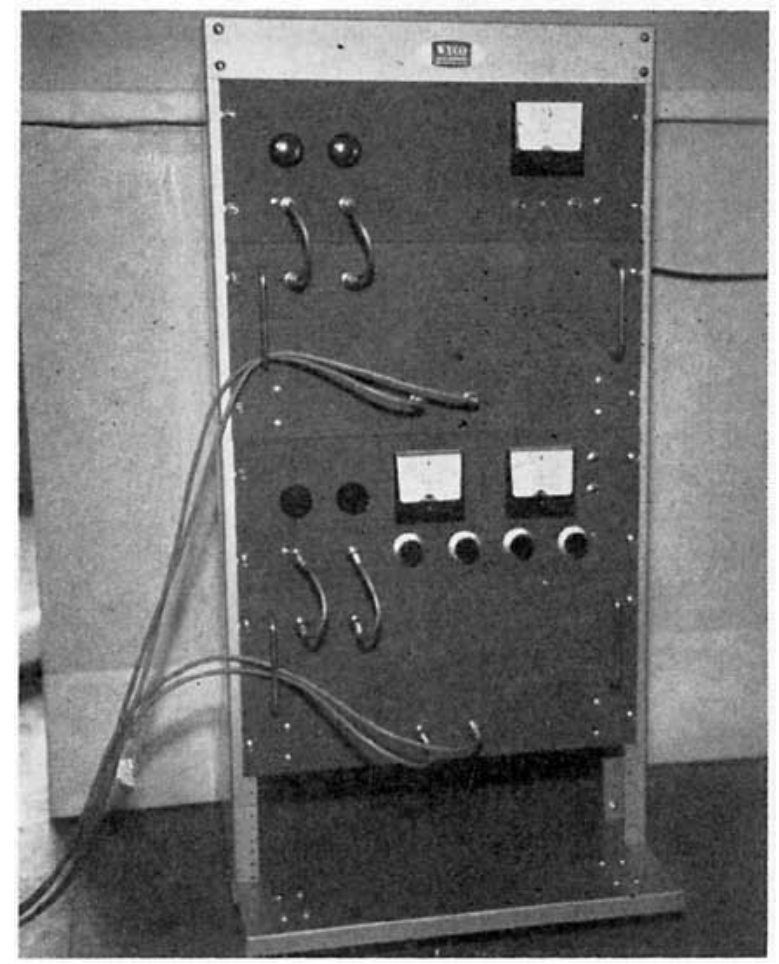

FIG. 2(a). Electronic unit of the sonic anemometer-thermometer.

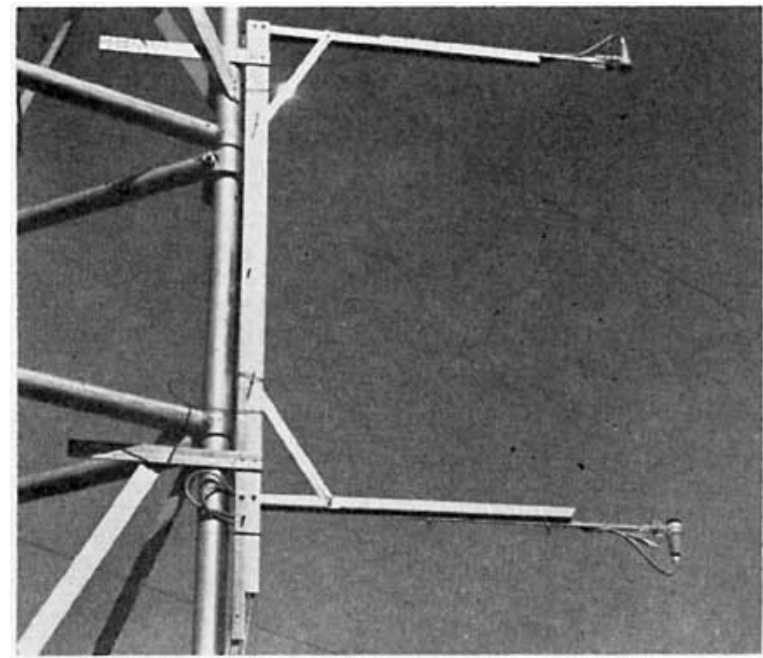

FIG. 2(b). Acoustic array mounted vertically for measuring $w^{\prime}$. 
tronic unit generates the two frequencies $f_{1}$ and $f_{2}$ and applies them to the corresponding transmitters. Signal frequencies picked up by the two receivers are amplified and their phases compared with that of reference signals obtained from the frequency generators. A summing network performs the operations $\left(\phi_{2}-3 / 4 \phi_{1}\right)$ and $\left(\phi_{2}+3 / 4 \phi_{1}\right)$ where $\phi_{1}$ and $\phi_{2}$ are phase angles in the two channels. These signals are subsequently recorded. The fluctuating parts of the outputs correspond according to (11) and (12) to the vertical wind and temperature fluctuations, respectively. A block diagram of the complete system is shown in Fig. 3.

The frequency standard (type 2001-2H, American Time Products) generates a signal with a frequency of $2500 \mathrm{cps}$. The frequency is stabilized through the use of a miniature tuning fork which is of bimetallic construction; the drive and pickup systems are electromagnetic. This frequency is multiplied three and four times, respectively, in the two frequency multipliers to obtain the 7.5-kc and $10-\mathrm{kc}$ signals used in the S. A. T.

The transducers in the acoustic array are temporary choices. Crystal earphones (Philmore No. 748) are used as transmitters and slim lavalier-type dynamic microphones (Olson M-137) as receivers. Much smaller transmitters and receivers are desirable, but for measuring vertical wind components with path length between $50 \mathrm{~cm}$ and $150 \mathrm{~cm}$, the above units are entirely adequate. Frequency response curves for a transmitter- receiver system with two configurations encountered in the acoustic array are shown in Fig. 4. Curve 1 shows the response of a receiver at the sonic output from a transmitter $67 \mathrm{cms}$ away and facing it. This represents the signal level at the receiver. Curve 2 shows the response of a receiver to the same sonic output from the transmitter only $2.5 \mathrm{cms}$ away from it but facing in the same direction as the receiver. It represents the level of the unwanted signal from the adjacent transmitter in the acoustic array. The input to the transmitter is maintained at 4 volts in both cases. The peaks of both curves lie in the vicinity of $6.2 \mathrm{kc}$ and the two frequencies, $7.5 \mathrm{kc}$ and $10 \mathrm{kc}$, lie on a slope. From the two curves, signal-to-noise ratio at the two receivers can be determined. (The term "noise" is used here rather loosely to mean the signal from the other channel.) The disparity in the ratios, $2.4 \mathrm{db}$ in the $7.5-\mathrm{kc}$ channel and $-33 \mathrm{db}$ in the $10-\mathrm{kc}$ channel, is a consequence of choosing frequencies on the slope of the response curves. By applying a larger signal voltage to the 10 -kc transmitter and reducing the signal to the $7.5-\mathrm{kc}$ transmitter, it is possible to achieve a more equitable distribution of signal-to-noise ratio in the two receivers.

Signal voltages for the high impedance crystal transmitters are obtained from a cathode follower output stage immediately following each frequency multiplier. The amplitude of the output signal can be adjusted to any value between 0 and 7 volts.

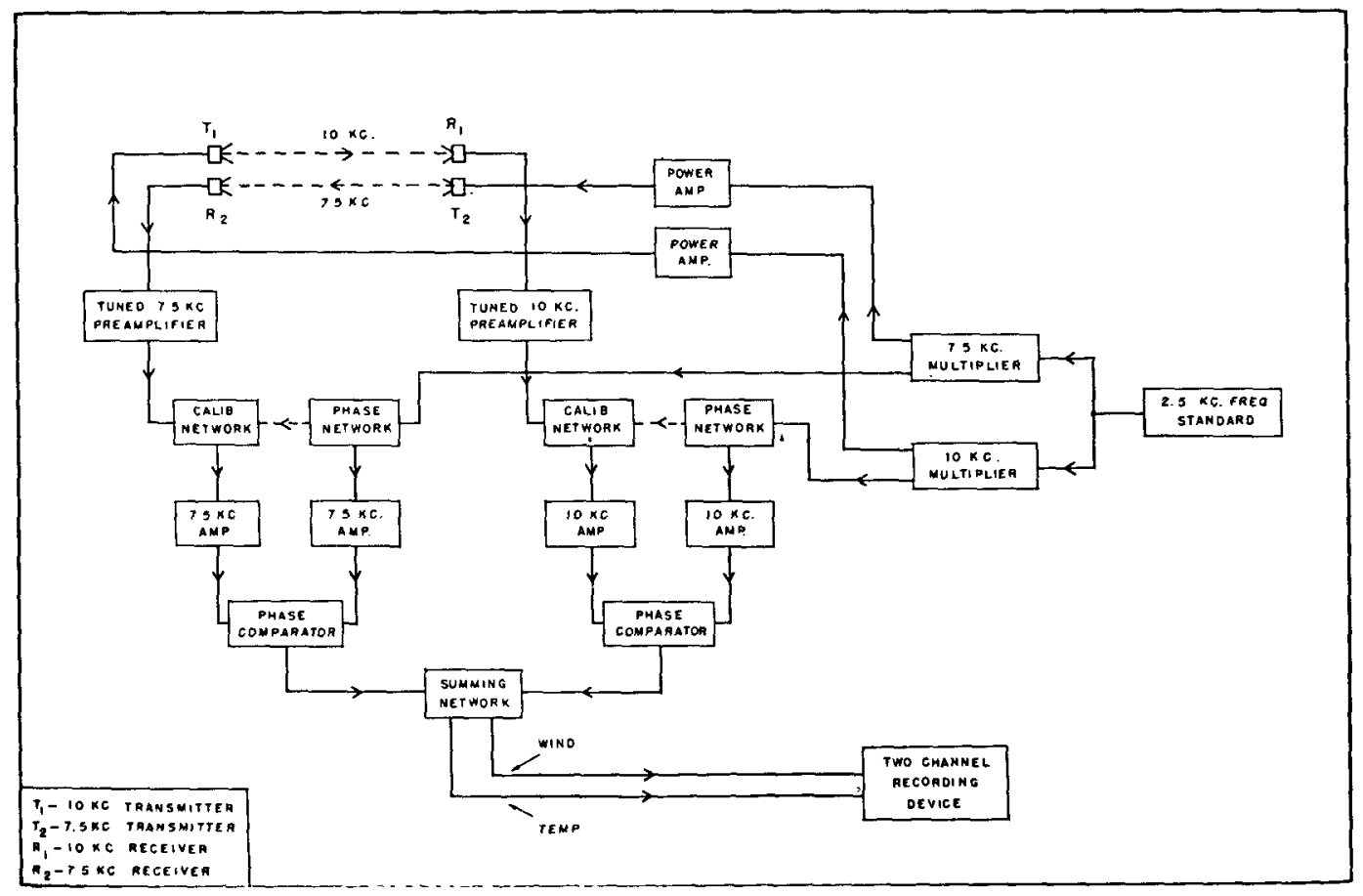

FIG. 3. Block diagram of sonic anemometer-thermometer. 
The output from each receiver goes directly into a preamplifier which has a band-pass filter tuned to the channel frequency and a rejection filter adjusted to further attenuate the noise frequency. Response curves for preamplifiers in both channels are shown in Fig. 5. The gain ratio of $64 \mathrm{db}$ between the channel and noise frequencies is necessary to offset the greater sound intensity of the undesired frequency at the receivers. Maximum gain at channel frequency is approximately $10^{5}$. If path distance is $1 \mathrm{~m}$ or less, full gain is not re-

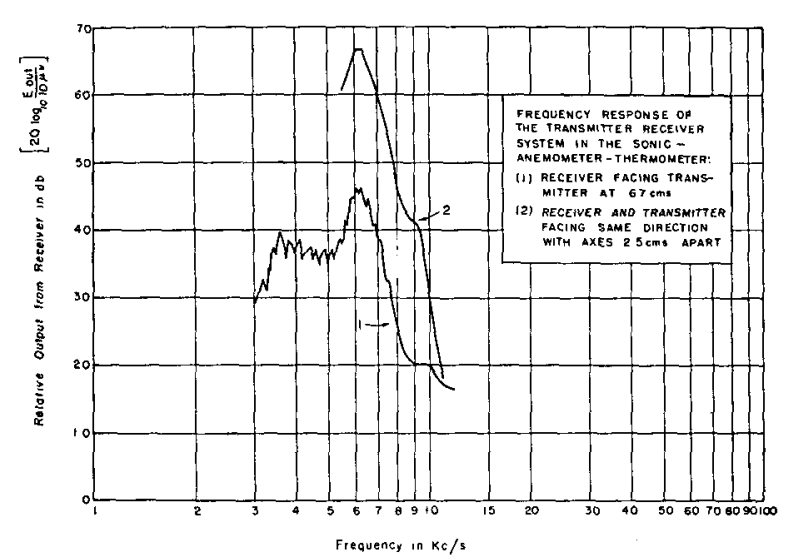

FIG. 4. Frequency response of the transmitter-receiver system. quired. The gain control in each preamplifier is set for one volt peak output signal.

For normal operation of the S. A. T. the preamplifier output is applied to the receiver amplifier through a calibration network; the reference signal (also one volt peak) from the corresponding frequency multiplier is applied to the reference amplifier through a phase-shift network. Switching arrangement in each calibration network enables the operator to substitute for the preamplifier output, fixed signals for calibrating the phase

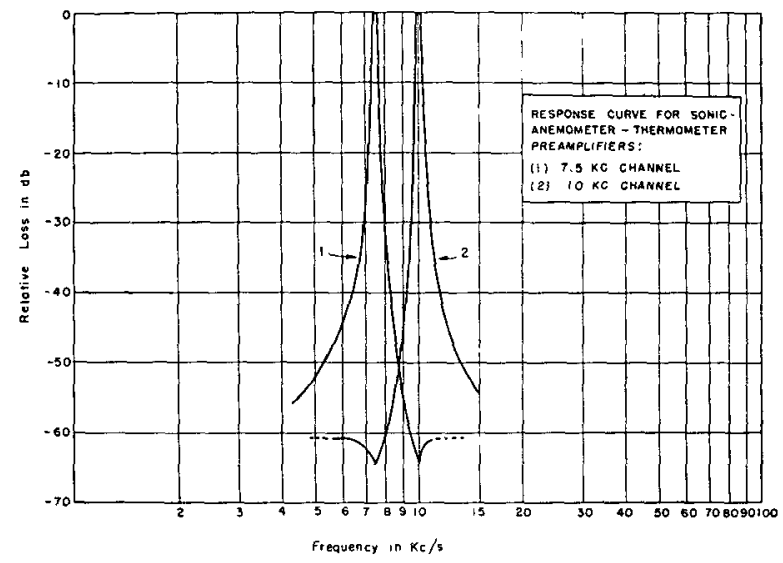

FIG. 5. Frequency response of the preamplifiers in the $7.5-\mathrm{kc}$ and $10-\mathrm{kc}$ channels.

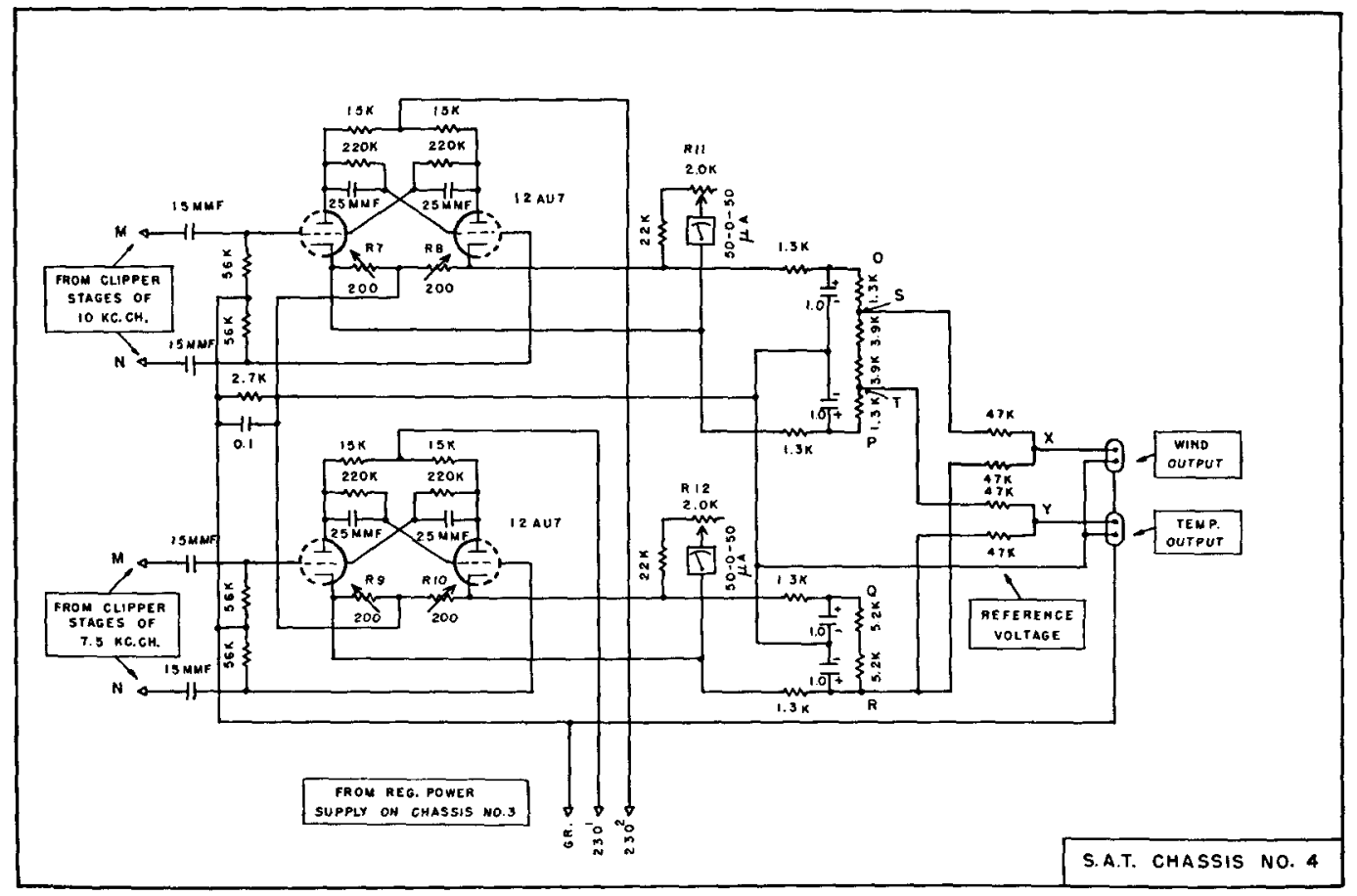

FIG. 6. Schematic circuit diagram showing multivibrator circuits in the phase comparators and summing network. 
comparators (in degrees angle) and the recording device (in cms per sec and degrees centigrade). The phase-shift network, on the other hand, provides the operator with a means for shifting the phase of the reference signal through a complete cycle. This enables him to set the initial mean phase difference in either phase comparator at any desired value.

The signals are amplified to approximately 100 volts by the receiver and reference amplifiers. In the phase comparators each of these signals is converted into a series of negative pulses; each negative pulse corresponds in time to the instant when the original sinusoid crosses the axis in the negative direction. Conversion of the sinusoids into pulses is achieved through the conventional technique of limiting, differentiating and clipping. Negative pulses derived from the received and reference signals are then applied to the grids of a bistable multivibrator, final stage of the phase comparator. The use of multivibrator circuits for phase comparison is well known, but the circuitry used and the coupling of these to the summing network are of particular interest.

When the two series of pulses are applied to the two inputs, $\mathrm{M}$ and $\mathrm{N}$, of a multivibrator circuit as shown in Fig. 6 (received side to $\mathrm{M}$ and reference side to $\mathrm{N}$ ), flip-flop action will proceed in such a way that one tube conducts for a period proportional to the phase difference between the received and reference signals, and the other tube conducts for the remainder of the period. Assuming perfect symmetry in the two sections, the potential difference at the output, i.e., between the two cathodes, takes the shape of a pulse-width modulated signal of constant amplitude. The width of the pulse varies from nearly zero (for $0-\mathrm{deg}$ phase difference) to almost the whole period (for 360-deg phase difference). At 180-deg phase difference the signal is a symmetric square wave. A D'Arsonval-type center-zero voltmeter connected across the cathodes will indicate the integrated value of the potential difference. Zero reading on the meter is therefore to be interpreted as 180-deg phase difference between the two input voltages. Full deflection to the left will correspond to 0 -deg difference and full deflection to the right to $360 \mathrm{deg}$. For phase angles between $0 \mathrm{deg}$ and $360 \mathrm{deg}$ the scale is continuous and linear. In practice, readings very close to $0 \mathrm{deg}$ and 360 deg are not possible because negative pulses arriving simultaneously at both sections can cause only one change of state, resulting in a square wave of half the original frequency. The meter, however, will indicate zero, which is also the reading for $180 \mathrm{deg}$. This ambiguity must be avoided; to do so, one must initially adjust the phase difference in the phase comparators as outlined in Section 4.

An unusual feature in the design of the multivibrator circuits is the use of a common cathode bias resistor. The cathode current in each multivibrator circuit is nearly constant except for switching transients which are absorbed by the cathode capacitor. The common bias resistor insures equality in the d.c. reference level of the outputs from both multivibrators, an essential requirement for the successful operation of the summing network. Variable resistors R7, R8, R9 and R10 make it possible to compensate for nonsymmetry in the two circuits. Each of these resistors is individually adjusted to produce exactly the same voltage drop (1.25 volts) across it when the corresponding section is conducting continuously. To cause continuous conduction in either section of a multivibrator one need only remove the negative pulses from the grid of that section; provision for removing the pulses individually from each of the four sections is made in the calibration switches. The phase indicating meters shown in Fig. 6 are first adjusted to give full-scale deflection in either direction for a potential difference of 1.25 volts. Then, with the aid of the calibration switches, each section is made to conduct continuously while the variable resistor in that section is adjusted accurately for full-scale deflection in the corresponding direction.

The summing network integrates the outputs from the phase comparators and computes from these the magnitude and direction of the fluctuations in wind and temperature. Addition and subtraction of the phase angles are possible because of the inverse nature of the wave forms at the two cathodes. A change in the average voltage at one cathode is accompanied by an equal and opposite change in the other cathode. The operation of the summing network may be illustrated by the following example: Assume that $E_{0}$ is the d.c. voltage at points $\mathrm{O}, \mathrm{P}, \mathrm{Q}$ and $\mathrm{R}$ (see Fig. 6) when the phase difference is 180 deg. If R7, R8, R9 and R10 are adjusted correctly, all these points will be at the same d.c. voltage with respect to the ground. Let a phase shift of $+\phi_{1}{ }^{\prime}$ in the $10-\mathrm{kc}$ channel raise the voltage at point $\mathrm{O}$ from $E_{0}$ to $\left(E_{0}+e_{1}\right)$. At $\mathrm{P}$ the voltage automatically drops to $\left(E_{0}-e_{1}\right)$. Similarly, a phase shift of $+\phi_{2}{ }^{\prime}$ in the $7.5-\mathrm{kc}$ channel changes the voltages at $\mathrm{Q}$ and $\mathrm{R}$ to $\left(E_{0}+e_{2}\right)$ and $\left(E_{0}-e_{2}\right)$, respectively. The wind and temperature outputs are obtained by measuring the voltages at points $\mathrm{X}$ and $\mathrm{Y}$ with respect to a reference voltage $E_{r}$ equal to the voltage drop across the common cathode resistor. Hence, the wind output will read $\left(E_{0}-E_{r}\right)$ $-1 / 2\left(e_{2}-3 / 4 e_{1}\right)$ and the temperature output, $\left(E_{0}-E_{r}\right)$ $-1 / 2\left(e_{2}+3 / 4 e_{1}\right)$. The advantage gained from measuring the outputs with respect to $E_{r}$ is a significant reduction in noise caused by fluctuations in the $\mathrm{B}^{+}$ voltage. There is, however, a small inconvenience caused by the fact that differential inputs are required in the recording device.

The front end of the recording system consists of a dual channel, low gain d.c. amplifier with input characteristics to match those of the S. A. T. outputs and with separate zero-suppression adjustments in each channel to compensate for the constant bias voltage $\left(E_{0}-E_{r}\right)$. The amplifier outputs are connected directly to an 


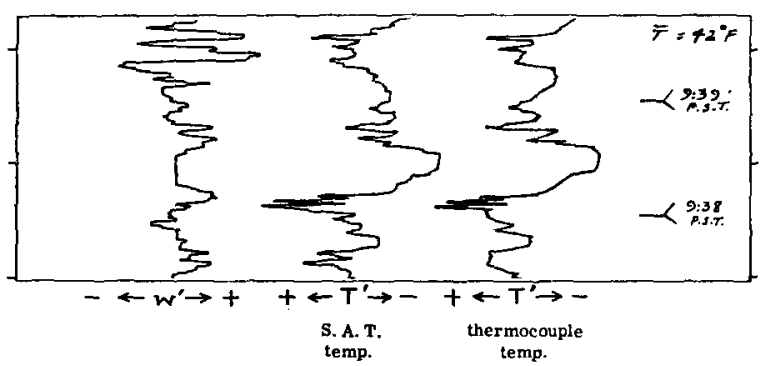

Fig. 7 (a). Temperature fluctuations of the sonic anemometerthermometer compared to reconstructed thermocouple record; the scales are obtained from Fig. 7 (b).

analog magnetic tape recorder (Tanberg Model C stereo recorder and two Mnemotron Model 102A converters) and a two-pen strip-chart recorder (Leeds and Northrup Speedomax G Model S recorder). Three standard speeds, $71 / 2,33 / 4$ and $17 / 8$ inches per sec, are available for recording and playback of the magnetic tape, but the strip-chart recorder has a fixed speed set at 1.1 inches per min. The frequency response of the analog tape recorder extends from d.c. to $200 \mathrm{cps}$, more than adequate for recording wind and temperature fluctuations in the atmosphere. The strip-chart recorder, while sluggish by comparison, performs the important function of providing a visual record of the data collected on tape.

\section{Observation technique}

For field measurements of vertical velocity and temperature fluctuations, the acoustic array is mounted vertically on a tower with the $10-\mathrm{kc}$ transmitter facing upward and the 7.5-kc transmitter downward. This particular arrangement is consistent with the development of $\mathrm{Eq}$ (11) and (12). A positive change in $w$ will cause an increase in the wind output voltage; a positive change in $T$, on the other hand, will cause a decrease in the temperature output voltage. The acoustic array may also be mounted in an inverted position, in which case the direction of $w$ on the chart will be reversed.

Before starting to record the fluctuations it is necessary to calibrate the chart paper in terms of velocity and temperature. This is done by first obtaining, in both channels of the recorder, a brief trace corresponding to $\phi_{1}=180 \mathrm{deg}, \phi_{2}=180 \mathrm{deg}$, and then suddenly changing $\phi_{2}$ to $150 \mathrm{deg}$, keeping $\phi_{1}$ the same. Both traces shift abruptly, as seen in Fig. 7(b). Putting $\phi_{1}^{\prime}=0$ deg and $\phi_{2}{ }^{\prime}=-30 \mathrm{deg}$ in (11) and (12), it is possible to determine what these shifts mean in terms of wind and temperature changes. Facility for making these shifts accurately is provided in the calibration switches. If, later on, the potentiometers in the attenuator circuits associated with the recorder are readjusted, the calibration procedure has to be repeated and a new calibration obtained for subsequent observations.

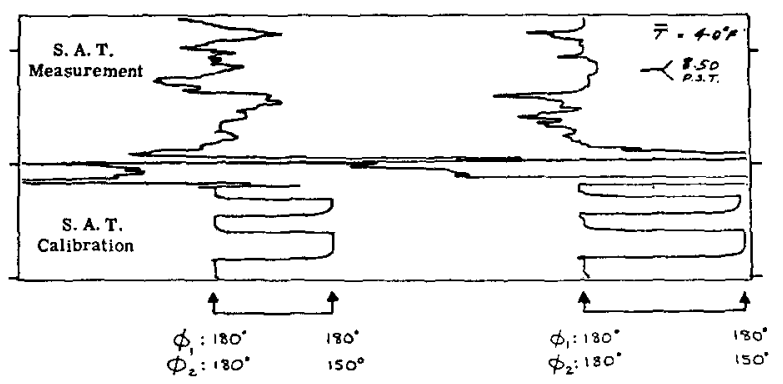

Frg. 7 (b). Calibration shifts made prior to recording Fig. 7 (a); the step changes correspond to $60 \mathrm{~cm} \mathrm{sec}-1$ in $w$ and $-1 \mathrm{C}$ in $T$.

When the calibration switches are turned to the position for sonic measurement, the preamplifier outputs are connected to the receiver amplifiers. The phase indicators will suddenly become alive and show fluctuations corresponding to phase shifts experienced by the sound waves picked up at the receivers. Each indicator needle will fluctuate about a mean phase reading. With the help of the phase-shifting controls this mean phase reading can be brought very close to $180 \mathrm{deg}$. The purpose of initially setting the phase angles at $180 \mathrm{deg}$ is to allow for the largest possible range of the wind and temperature fluctuations. It must be stressed here that the initial phase setting has no effect on the accuracy of the instrument as long as the fluctuations stay clearly within the linear range of both phase comparators. The operator of the S. A. T. may choose other initial settings for a specific purpose. For example, if a large rise of mean temperature $(7-8 \mathrm{C})$ is expected during the run it would be necessary to set both channels initially at about $90 \mathrm{deg}$. If, on the other hand, a similar drop in the mean temperature is anticipated, the setting in both channels should be roughly $270 \mathrm{deg}$.

\section{Preliminary field tests}

In the development of an absolute instrument like the S. A. T. the designer relies heavily on the physical principles upon which its theory is based. Unless another more reliable method exists for verification, the precision and stability of the instrument have to be determined from a careful analysis of the total system. Indirect checks are then necessary in order to establish confidence in the instrument.

With the continuous wave approach used in the S. A. T. it is found that acoustic reflections cause significant error when the acoustic array is placed in an enclosed space such as a wind tunnel or a crude anechoic chamber. For this reason experiments with controlled environment have not yet been performed. The authors found that in the open air, reflections from the ground surface are negligible at heights greater than about $2 \mathrm{~m}$. No difference was observed when the ground below the acoustic array was covered with sound absorbing ma- 
terial. The effect of reflections was also tested by following the temperature trace after sunset when an inversion starts building upward from the ground. A drift in the temperature corresponding to variations in the phase of the reflected signals should occur as the air near the ground undergoes rapid cooling. S. A. T. temperature records for such a period have been compared very closely with temperature profile measurements made with thermocouples. There is no evidence of any such drift; agreement with temperature readings at the S.A.T. level is in fact remarkably good.

Two field tests of the S. A. T. were conducted at the experiment site of the Atmospheric Physics Operation, Hanford Laboratories, in eastern Washington. For both tests the acoustic array was mounted vertically on a portable tower with its midpoint $3.5 \mathrm{~m}$ above the ground. The tower itself was equipped for measuring horizontal winds and temperatures at 0.75-, 1.5-, 3-, 6-, 12- and 24-m heights.

The first field test was made primarily to evaluate the performance of the instrument. Since some knowledge exists about the behavior of turbulence in the atmospheric boundary layer, it was hoped that a detailed study of data from an extended run would provide some useful information concerning the instrument. $w$ and $T$ fluctuations under different meteorological conditions were first carefully examined; fluctuation patterns obtained were in good agreement both in their magnitude and structure with patterns expected for the particular conditions. Quantitative estimates were then made of heat flux by eddy correlation method for extreme lapse and inversion conditions. The estimates appear very reasonable, and for the lapse condition agreement with Priestley's (1959) heat flux equation is remarkably good.

A direct check on the S. A. T. temperature measurement was made by comparing it with a measurement made with a thermocouple half way between the transmitting heads. Fig. 7(a) shows a portion of the thermocouple record reconstructed to match the scale and chart speed of the S. A. T. record. Agreement is again very good, considering the fact that the $S$. A. T. temperature is the integrated value over the length of the acoustic path, while the thermocouple represents a point measurement. Actual magnitude of the fluctuations can be determined from the calibration traces in Fig. 7(b). A path length of $103 \mathrm{cms}$ was used for all runs in this first series of tests and all the recordings were made on strip chart.

In the second field test, the path length was reduced to $50 \mathrm{~cm}$ to take advantage of the better response characteristics of the newly added analog tape recorder. Recordings were made simultaneously on magnetic tape (at $71 / 2$ inches per sec) and on strip chart. The tapes were later played back into the strip chart recorder at $17 / 8$ inches per sec. As expected, the smaller acoustic path length, together with the expanded time scale, revealed some of the finer details in the fluctuations. It was thus possible to examine very closely the structure of convective elements and to develop a simple model based on the distribution of $w^{\prime}$ and $T^{\prime}$ within the element. This and the results from the first field test are described in another paper in this Journal of Applied Meteorology. (See pages 180-186.)

\section{Limitations in accuracy}

Four types of errors are possible in this instrument. They occur

(a) from approximations made in the theoretical development,

(b) within the instrument,

(c) from external causes such as random noises, reflections, etc.,

(d) from the operator.

The most serious error from approximations in theory occurs in the temperature measurement and is caused by the humidity and horizontal velocity terms in (9). The humidity error can be neglected for values of $\beta$ larger than 2, as was the case at the Hanford tests. The figure may not be so low for measurements over water or over wet land, and appropriate correction should be made in the temperature calibration. The error due to horizontal velocity is very small (approximately 0.2 per cent) for a speed of $1 \mathrm{~m} \mathrm{sec}^{-1}$ but increases almost quadratically with speed and becomes serious for wind speeds in excess of $5 \mathrm{~m} \mathrm{sec}{ }^{-1}$ when $u^{\prime}$ is large. Another error which can affect both $w^{\prime}$ and $T^{\prime}$ measurements is due to changes in $\bar{T}$, and therefore in $\bar{C}$ with the time of day. An error of about 0.3 per cent in $w^{\prime}$ and about 0.5 per cent in $T^{\prime}$ may be introduced with every degree Centigrade change in ambient temperature. 'This error can be minimized by making new calibrations at hourly intervals during operation.

The next type of error is hard to determine exactly. Precautions have been taken in the design of the instrument to reduce such errors. The frequency standard is accurate to within 0.001 per cent and has a temperature coefficient of less than one part per million per degree Centigrade. Regulated power supplies are used in all circuits; the signal-to-noise ratio at the outputs is improved by measuring them with respect to the reference voltage $E_{r}$. The noise generated within the instrument is low-on the order of $0.5 \mathrm{~cm} \mathrm{sec}-1$ at the wind output, and $0.01 \mathrm{C}$ at temperature output for $d=1 \mathrm{~m}$. A serious error would be introduced if the variable resistors R7, R8, R9 and R10 were not correctly adjusted. These adjustments are within the control of the operator and can easily be made accurate to within 0.4 per cent. A similar error is caused by inequalities in the resistors used in the summing network. The resistors have been carefully chosen from one per cent precision resistors and the largest error possible in this circuit is 1.5 per cent. A systematic error is caused by inaccuracy in the 
30-deg calibration shift, but this error can be kept within one per cent if proper care is exercised in adjusting the calibration networks. Variations in the amplitude of the received signals due to air turbulence, which cause significant error in the pulse type sonic anemometer, did not have any detectable effect on the wind and temperature measurements.

It has been observed that random noises such as human speech, automobile noises, etc., have little effect on the performance of the instrument. High-pitched noises like the jingling of keys do cause interference, but such sounds are not likely to be encountered in the field. Another form of extraneous noise would arise from reflections of the transmitted signals from large, flat surfaces in the vicinity of the instrument. Experimental sites chosen for micrometeorological observations are usually open areas where the earth is the only significant reflector. The test described in Section 5 shows that above a height of $2 \mathrm{~m}$ the reflected wave is too small to be of any consequence at the frequencies used in the S. A. T.

The last type of error is caused by the operator in installing the acoustic array, measuring the acoustic path length, and determining $\bar{T}$, all of which together can easily be kept within one per cent.

Based on the foregoing analysis, maximum errors of 4 per cent in the vertical wind and 5 per cent in the temperature have been computed for measurements made at Hanford. The maximum possible error in the determination of the eddy heat flux is thus within 10 per cent.

A limitation in the S. A. T. with no bearing on its accuracy is the degradation in its frequency response due to the automatic spatial integration along the acoustic path. For eddy diameters smaller than the path length, attenuation will be severe. Since eddies tend to move with the mean wind, the corresponding eddy frequency becomes a function mainly of the horizontal wind speed. For example, with a horizontal wind speed of $5 \mathrm{~m} \mathrm{sec}^{-1}$ and a path length of $50 \mathrm{~cm}$, the cut-off frequency is approximately $10 \mathrm{cps}$. Realization of this characteristic is important when other types of measurements are made for use in conjunction with the S. A. T. data. Thus, for humidity measurements to compute latent heat flux or to provide compensation for humidity error in the S. A. T. temperature, an open path infrared hygrometer (with same path length as the S. A. T.) would seem a most appropriate choice.

Acknowledgments. We are indebted to Mr. P. Schofer of the University of Wisconsin for the initial idea from which the S. A. T. has emerged. We wish to thank Messrs. J. J. Fuquay, C. E. Elderkin and P. W. Nickola at the Atmospheric Physics Operation, Hanford Laboratories, for their generous assistance during the field experiments.

\section{REFERENCES}

Barret, E., and V. E. Suomi, 1949 : Preliminary report on temperature measurements by sonic means. J. Meteor., 6, 273-276.

Bovsheverov, V. M., and V. P. Voronov, 1960: Acoustic anemometer. Izvestia Geophys. Series, 6, 882-885.

Corby, R. F., 1950: Acoustic anemometer-anemoscope. Electronics, 23 (1), 88-90.

Gurvich, A. S., 1959: Acoustic microanemometer for investigation of the microstructure of turbulency. Acousitics $J$. (USSR), 5 (3), 368-369.

Priestley, C. H. B., 1959: Turbulent transfer in the lower atmosphere. Chicago, Univ. Chicago Press, 130 pp.

Schotland, R. M., 1955: Measurement of wind velocity by sonic means. J. Meteor., 12, 386-390. 